\title{
Supporting Information: All-Optical Wavelength Conversion by Picosecond Burst Absorption in Colloidal PbS Quantum Dots
}

\author{
Pieter Geiregat, ${ }^{*,+, \uparrow}$ Arjan Houtepen, \\ Hens ${ }^{*,+,}, \mathbb{9}$ \\ $†$ Photonics Research Group, Ghent University, Belgium \\ $\ddagger$ Physics and Chemistry of Nanostructures, Ghent University, Belgium \\ I Center for Nano and Biophotonics, Ghent University, Belgium \\ $\S$ Opto-Electronic Materials Section, TU Delft, The Netherlands \\ E-mail: Pieter.Geiregat@UGent.be; Zeger.Hens@UGent.be
}

\section{S1 Spectral Shifts}

A transient absorption spectrum can be interpreted in terms of different effects, which include (1) the blocking of electronic transitions due to state filling - typically resulting in a negative signal or bleach,$-(2)$ the opening of new electronic transitions, which typically leads to photoinduced absorbance and (3) a shift of the linear absorption spectrum due to, e.g., repulsive/attractive interaction of multi-excitons, ${ }^{1}$ which can lead to either a reduction or an increase of the absorbance. When the transient absorption spectrum is only due to a spectral shift $\delta E$, the latter can be readily determined from the ratio between the transient absorption $\Delta A$ - defined as the difference $A^{\star}-A_{0}$ between the absorbance after and before photoexcitation - and the derivative of the linear absorption spectrum provided that $\delta E$ is 

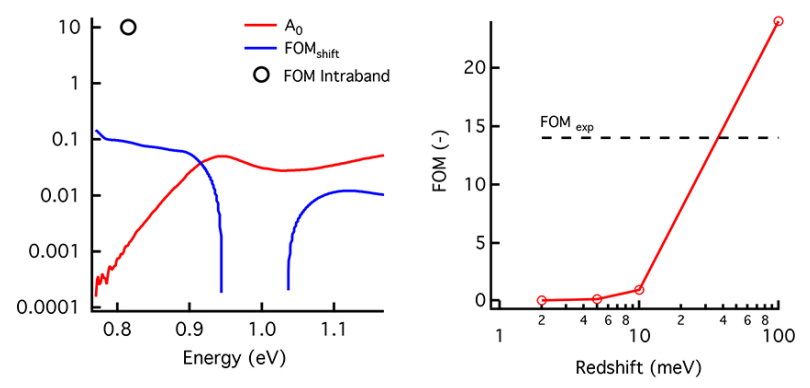

Figure S1: Influence of shifts on FOM: (left) Linear absorption $A_{0}$ versus energy together with the predicted FOM based on Equation S1 for a shift $\langle N\rangle \times \delta_{E}=3 \mathrm{meV}$. The experimentally observed FOM for $\langle N\rangle=1$ is indicated with an empty circle. (right) The FOM at the matching wavelength calculated using only the spectral shift as indicated on the horizontal axis. One can view this as similar to $3 \mathrm{c}$ as the overal shift can be written as $\langle N\rangle \times \delta_{E}$, with $\delta_{E}$ the shift per exciton created.

sufficiently small: ${ }^{1}$

$$
\delta E=\frac{A_{0}\left(E_{0}\right)-A^{\star}\left(E_{0}\right)}{\left.\frac{\partial A_{0}}{\partial E}\right|_{E=E_{0}}}=-\frac{\Delta A\left(E_{0}\right)}{\left.\frac{\partial A_{0}}{\partial E}\right|_{E=E_{0}}}
$$

\section{S2 Synthesis of PbS nanocrystals}

Oleylamine (OLA) capped PbS QDs were synthesized using the procedure described by Cademartiri19 and modified by Moreels et al.. ${ }^{2}$ After synthesis, the OLA ligand shell is substituted by oleic acid (OA). An exchange to OA is typically performed by adding OA to a toluene suspension of $\mathrm{PbS}$ QDs in a ratio of 1.5:10 OA/toluene After precipitation with ethanol and centrifugation, the QDs are re-suspended in toluene and the exchange is repeated twice until final dissolution in hexane. Thin films are fabricated using spincoating from a dilute solution at $2000 \mathrm{rpm}$ on silica glass substrates. Film thickness is obtained from AFM analysis and optical measurements. 


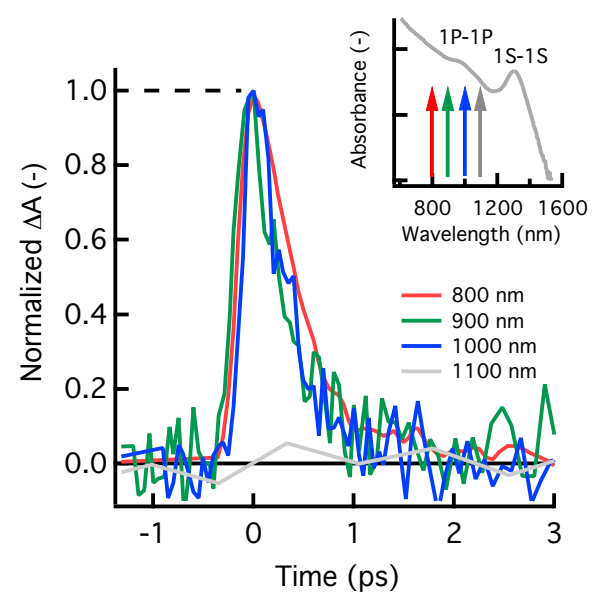

Figure S2: Normalized transients at the matching wavelength for different pump wavelengths.

\section{S3 The Pump Wavelength Dependence}

By varying the pump wavelength, we tried to estimate a cut-off for the conversion scheme. While pumping at wavelengths shorter than the 1P-1P transition (at around $1050 \mathrm{~nm}$ ), we observe similar dynamics throughout the range from 700 to $1000 \mathrm{~nm}$ (see Figure S2). Pumping at wavelengths beyond the $1 \mathrm{P}-1 \mathrm{P}$ manifold, no return-to-zero transients are observed. This corresponds quite well to the idea of continuum cooling followed by a $1 \mathrm{P}$-to-1S limiting step where indeed carriers are only 'hot' (and therefore inducing the hot carrier intraband absorption during cooling) when their excess energy exceeds the 1P-1S separation.

\section{S4 Multi-Pulse Generation}

To analyze the response of the sample under multi-pulse excitation (so-called 'pulse trains'), we employed a pump-repump technique. As explained in the Methods section, this allows us to create an arbitrary bit pattern. The idea is based on the difference in group velocity between ordinary (o) or extra-ordinary (e) polarized beams going through a birefringent crystal. We use $\mathrm{YVO}_{4}$, a positive uniaxial crystal with $n_{e}>n_{o}$, commercially available through e.g. Altechna. 
In general, we can decompose a linearly polarized pulse going through such a crystal as a superposition of two orthogonally polarized pulses, one along each of the special crystal axes (orindary and extra-ordinary). The difference in group delay causes, if sufficiently large, the pulses to dissociate in time. As such, we are left with two orthogonally polarized pulses with controlled temporal separation (2.2 ps for a single crystal in our case). These are then sent through a depolarizer to avoid any polarization effects.

The initial energy of the pulse $E_{p}$ is redistributed over both pulses according to a simple $\cos ^{2}$-dependence, allowing us to write for example for the energy in the second pulse $E_{2}$ :

$$
E_{2}=E_{p} \times \cos ^{2}\left(\theta-\theta_{\text {axis }}\right)
$$

where $\theta$ is the angle of the initial polarization and $\theta_{\text {axis }}$ is the angle of the ordinary axis of the birefringent crystal. As such, we can tune the energy distribution over both pump pulses continuously. The effect on the observed FOM per pulse is shown in Figure S3. The retreived $\cos ^{2}$-dependence indicates that the transfer from input (the pump pulse sequence) to output (the modulation on the probe) is indeed linear. A similar reasoning holds for the case of 4 pulses, where we employ 3 crystals to obtain: first 2 crystal create a separation of $4.4 \mathrm{ps}$, where the third crystsal splits those again to create 4 pulses separated by $2.2 \mathrm{ps}$.

Figure S4 shows the relative FOM for varying input pump beam polarization going through the birefringent $\mathrm{YVO}_{4}$ crystal. A clear cosine dependence is observed which corresponds to the predicted energy distribution over both ordinary and extra-ordinary pulses.

\section{S5 The Intrinsic Absorption Coefficient}

The absorption coefficient $\mu$ of a material is related to its absorbance $A$ as:

$$
\mu=\frac{\ln (10) A}{L}
$$




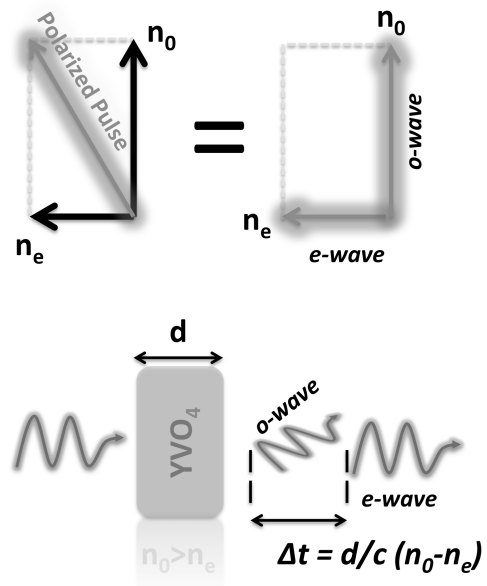

Figure S3: A linerarly polarized pulse in the framework of a birefringent crystal can be seen as a superposition of an extra-ordinary wave (e) and an ordinary wave (o), where both experience different indices of refraction and therefore different group velocities. This effect leads to a separation of the initial pulse in time proportional to the index difference $\left|n_{e}-n_{o}\right|$ times the crystal length. In the case of $1 \mathrm{~mm}$ thick $\mathrm{YVO}_{4}$ crystals

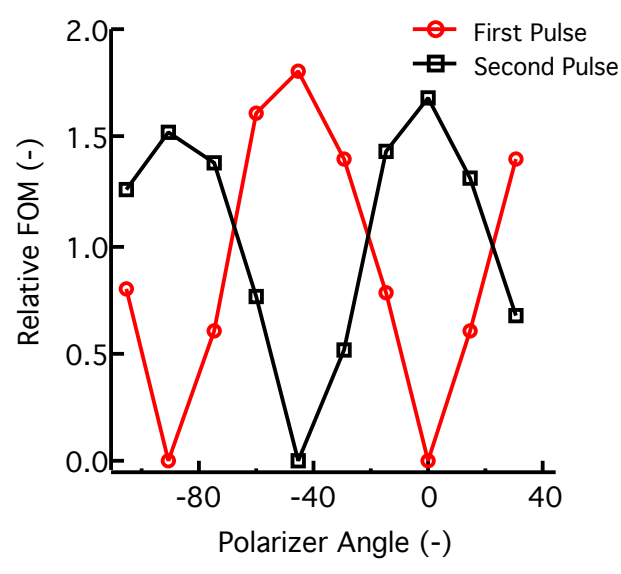

Figure S4: Relative FOM for varying input pump beam polarization going through the birefringent $\mathrm{YVO}_{4}$ crystal.

Here, $L$ is the interaction length (e.g. the length of the cuvette containing a dispersion or the thickness of a film. Note that this relation also holds for the transients, i.e., $\Delta \mu \sim \Delta A$, since $\mathrm{L}$ is constant. In the case of a composite materials consisting of a absorbers embedded in a host - such as quantum dots in a dielectric medium - the intrinsic absorption $\mu_{i}$ is defined as the ratio between the absorption coefficient and the volume fraction $f$ of the absorbers: $\mu_{i}=\frac{\ln (10) A}{f L}$. Defining $\Delta \mu_{i}$ accordingly as the change of the intrinsic absorption coefficient 
under the condition $\langle N\rangle=1$, we arrive at the relation expressed by the equation in the main text.

\section{S6 Switching Energy Calculation}

To evaluate the energy required to convert a pump pulse with energy $E_{p u l s e, c}$ (at $\lambda_{c}$, e.g. at $1000 \mathrm{~nm})$ to a target wave at $\lambda_{t}($ e.g. $1520 \mathrm{~nm})$, we consider a standard slot waveguide configuration where the nanocrystals (QDs) are confined in an area S with transverse modal field confinement $\Gamma$, i.e. the fraction of the total optical mode that actually propagates in the area where the QDs are present. For simplicity, we only consider losses from the QD film, not from scattering or in and outcoupling to the waveguide itself. The intrinsic modulation scales with the average exciton density (see Figure 3) $\langle N\rangle$, created by absorption of photons at $\lambda_{c}$. Due to absorption of the carrier wave, the initial pulse energy will diminish upon propagation through the device with length $L$, such that in general $\langle N\rangle$ will be a function of position $x$ :

$$
\langle N\rangle(x)=J_{p h, c}(x) \times \sigma_{c}
$$

Here $J_{p h}(x)$ represents the photon flux per pulse at the carrier wavelength (photons $/ \mathrm{cm}^{2}$ ) and $\sigma_{c}$ is the absorption cross section of the QDs at that same wavelength. We can write $J_{p h, c}$ as follows:

$$
J_{p h, c}(x)=\frac{E_{p u l s e, c}}{\hbar \omega_{c}} \times e^{\left(-\Gamma f \mu_{i, c} x\right)} \times \frac{\Gamma}{S}
$$

where indices $(c, t)$ refer to (carrier,target) respectively. By definition (see further), the change in absorption scales with the average exciton density $\langle N\rangle$ through the intrinsic modulation coefficient $\Delta \mu_{t, i}$ :

$$
\Delta \mu_{t}(x)=\langle N\rangle(x) \times f \Delta \mu_{i, t}
$$

where $\Delta \mu_{i, t}=5200 \mathrm{~cm}^{-1}$. As such, we can write the following for the beam intensity $I$ at 
the target wavelength:

$$
\frac{d I(x)}{d x}=-\Gamma\left(\mu_{t}+\Delta \mu_{t}(x)\right) \times I(x)
$$

where $\Gamma \mu_{t}$ is the regular linear absorption loss at the target wavelength $\lambda_{t}$ without the carrier wave present. Solving this with the appropriate boundary conditions, gives us the excitinction of the target wave after propagating through a length $L$ in the presence of a carrier wave with energy $E_{p u l s e, c}$.

$$
\ln \left(\frac{I(L)}{I_{0}}\right)=-\left\{\Gamma f \mu_{i, t} L+\Delta \mu_{i, t} \times \frac{E_{p u l s e, c}}{\hbar \omega_{c}} \times \frac{\sigma_{c} \Gamma}{S \times \mu_{i, c} L} \times\left(1-e^{-\Gamma \mu_{i, c} f L}\right)\right\}
$$

Note that the attenuation of the target wave in absence of the pump pulse, is given by:

$$
\ln \left(\frac{I(L)}{I_{0}}\right)=-\Gamma f \mu_{i, t} L
$$

Remembering the expression for the FOM, we can rewrite the FOM based on the previous expression:

$$
F O M=\frac{\Delta \mu_{i, t}}{\mu_{i, t}} \times \frac{E_{p u l s e, c} \sigma_{c}}{S \hbar \omega_{c}} \times \frac{1-e^{-\Gamma f \mu_{i, c} L}}{f \mu_{i, c} L}
$$

To evaluate the energy required to switch with a given FOM, we can write this as:

$$
E_{p u l s e, c}=F O M \times \frac{\mu_{i, t}}{\Delta \mu_{i, t}} \times \frac{\hbar \omega_{c} S}{\sigma_{c}} \times \frac{f \mu_{i, c} L}{1-e^{-\Gamma f \mu_{i, c} L}}
$$

\section{S7 Return to zero}

The importance of return-to-zero after 1-2 ps is clearly illustrated in Figure S6 where transients at the matching wavelength $\lambda_{m}$ are compared to transient at wavelengths longer than $\lambda_{m}$ 

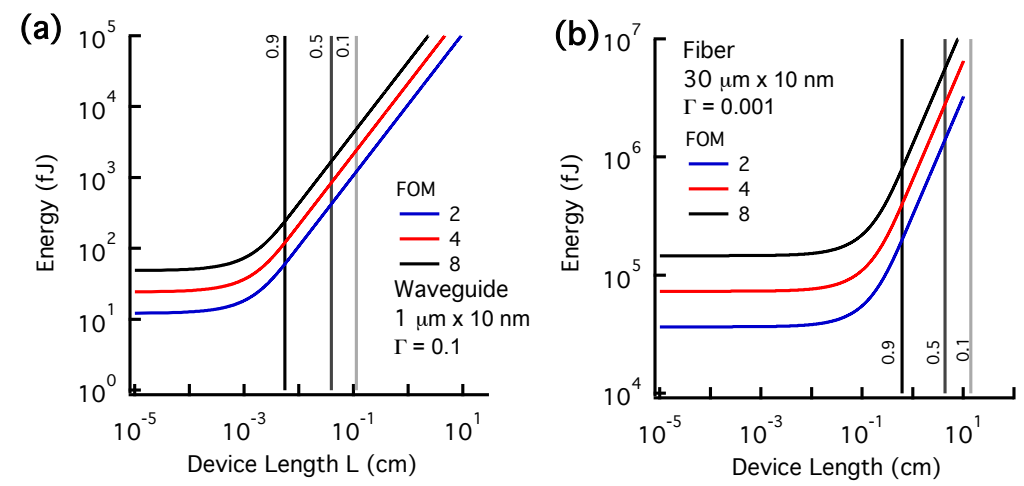

Figure S5: Evaluation of switching energy per bit (in fJ/bit) for a FOM of 1/2 (red) and 2 (blue) as function of device length (in $\mathrm{cm}$ ). The cases of a slot waveguide and an optical fiber are considered. The vertical lines correspond to linear extinctions in absence of the pump pulse of $0.9,0.5$ and 0.1 .
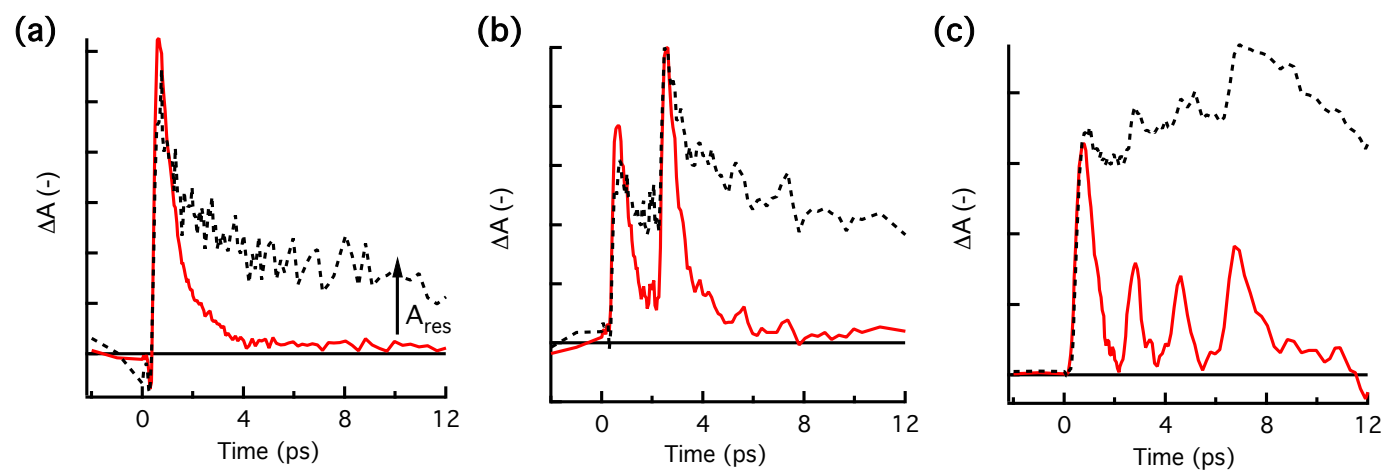

Figure S6: Transients at the matching wavelength (red) and a detuned wavelength $-\lambda_{m}+$ $20 \mathrm{~nm}$ - (black dashed) for single pulse, double pulse and multi-pulse experiments.

\section{S8 Contribution of Spontaneous Emission to FOM}

When the pump pulse (sequence) generates band edge excitons, these excitons can recombine radiatively over the course of time, which would lead to a negative contribution to $\Delta A$ : light emission adds photons to the beam or equivalently, reduces the absorbance of that beam. In principle, this effect could reduce the FOM or at least give an additional background. Let us consider the case where $\langle N\rangle=1$, which gives a FOM of 23. In this case, all dots are excited and will give rise to photon emission, characterized by a radiative lifetime of several microseconds. ${ }^{3}$ If we consider a single time-interval of 2 picoseconds, the probability to add a photon to the beam is $210^{-6}$ (or equivalently $10^{6}$ emission events per second per 
dot). Furthermore, photons are, due to inhomogeneous (size dispersion) and homogeneous (phonon coupling) effects, emitted in a broad band of ca. $200 \mathrm{~nm}$ (typical value for PbX $\mathrm{QDs}^{3}$ ) with a quantum yield smaller than unity. As the matching wavelength range is much smaller than this $200 \mathrm{~nm}$, the amount of photons actually contributing to the signal at $\lambda_{m}$ is even smaller than considered before by another 2 orders of magnitude. Finally, spontaneous emission is random and only a small fraction will couple to the right direction, i.e. the propagation direction of the pump and probe beams. As such, we can estimate that about $10^{4}$ emission events take place per QD per second. This number will be even lower due to the finite quantum yield and re-absorption by other QDs.

To estimate the number of absorption events per QD we can make use of the differential cross section, $\Delta \sigma=\Delta \mu_{i} \times V_{Q D}$ with $V_{Q D}$ the volume of the $4.3 \mathrm{~nm} \mathrm{PbS}$ QDs. We find a value of $2.110^{-16} \mathrm{~cm}^{2}$. If we assume a CW power level in the target/probe beam of $1 \mathrm{~mW}$, which is a reasonable level in modern optical telecommunication, we can estimate based on the area of the relevant optical mode (fiber or waveguide) what the number of absorption events per second is. In a $10 \mu \mathrm{m}$ core fiber (typical single mode fiber) with a modal confinement factor of unity, we find $410^{5}$ absorption events per second. For a waveguide $(10 \mu \mathrm{m} \times 10$ $\mathrm{nm})$ with unity modal confinement, we find $410^{8}$ absorption events per second. Both are orders of magnitude larger than the emission events (per second per dot), and as such we can neglect the contribution of spontaneous emission to the FOM.

\section{References}

1. Geiregat, P.; Houtepen, A.; Justo, Y.; Grozema, F. C.; Van Thourhout, D.; Hens, Z. Coulomb Shifts upon Exciton Addition to Photoexcited PbS Colloidal Quantum Dots. J. Phys. Chem. C 2014, 118, 22284-22290.

2. Moreels, I.; Lambert, K.; Smeets, D.; De Muynck, D.; Nollet, T.; Martins, J. C.; Van- 
haecke, F.; Vantomme, A.; Delerue, C.; Allan, G. et al. Size-dependent optical properties of colloidal PbS quantum dots. ACS Nano 2009, 3, 3023-30.

3. Justo, Y.; Geiregat, P.; Hoecke, K. V.; Vanhaecke, F.; Donega, C. D. M.; Hens, Z. Optical Properties of PbS / CdS Core / Shell Quantum Dots. J. Phys. Chem. C 2013, 117, 20171-20177. 